Terrestrial

\section{primordial neon}

SIR - In their letter ${ }^{1}$ about neon and helium isotopes at Loihi and Kilauea (Hawaii), Honda et al. published data similar to ours. But their interpretation is not as strongly supported by their data as they claim. In their Fig. 1, Honda et al. ${ }^{1}$ plot many more data than listed, and most of the data are indeed atmospheric within error. The $L-K$ correlation line is established using all of the data. One consequence is to force the regression line through the atmospheric point. But in the table, they removed those data that are atmospheric within two standard deviations, hence discarding both the data with large errors, but also those with small errors which plot close to the atmospheric composition.

Their Fig. 1 shows only six anomalous points, meaning that they plot above the mid-ocean-ridge basalt (MORB) area established by us $^{2}$, most of which have large errors. Moreover, consideration of only these points shows that they can be fitted by a line parallel to the mass fractionation line $(\mathrm{mfl})$. Mass fractionation is thus likely, and further confirmed by considering the duplicate analyses for samples KK29-4 and KK1712: the data are clearly different for both aliquots, and precisely plot on lines parallel to the mass discrimination line. This is shown in Fig. 1 here, where we plot total-gas data for the eight samples in the table of Honda et al. It is important to realize that mass fractionation can operate on non-atmospheric gas (see, for example, ref. 3).

The rest of the dispersion can be accounted for by mixing between

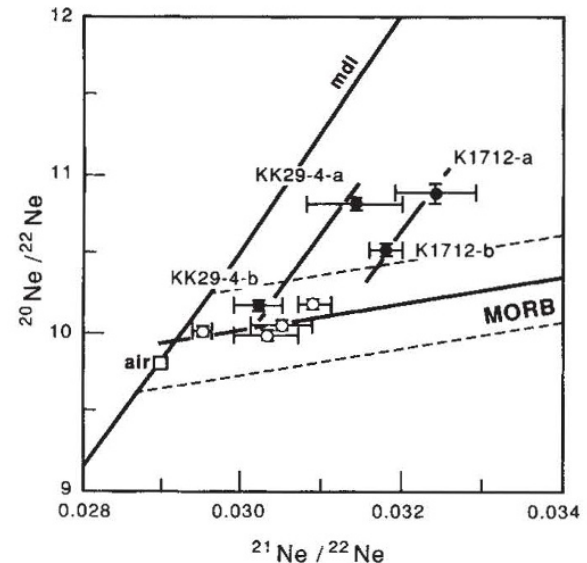

FIG. 1 Total gas neon data for all samples listed in ref. 1, plotted in the three-isotope neon diagram. Filled circles represent two duplicate analyses for samples KK29-4 and Kilauea 1712. The duplicate data lie on lines parallel to the mass discrimination line (mdl). Mixing is especially manifest for samples represented by the empty circles, while mass discrimination is likely for samples represented by the filled circles.

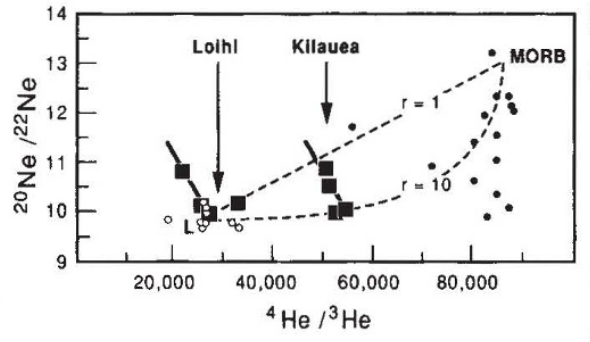

FIG. 2 Helium-neon isotope diagram. Filled circles, data for MORB; open circles, data for Loihi from ref. 2; squares, data for Loini and Kilauea from ref. 1. Mixing plus mass fractionation is manifest, especially for samples from Kilauea. Broken lines, mixing curves.

atmospheric-like neon (from the undegassed mantle) and degassed mantle-like neon (MORB), as for five points in our Fig. 1, which plot within the MORB area. Mixing has already been observed at Hawaii by Kurz et al. ${ }^{4}$, who used helium isotopes. For Loihi, mixing may be due to MORB neon from xenocrysts, present in alkali Loihi samples ${ }^{5}$, and shown to release MORB helium upon heating. We showed ${ }^{2}$ that the anomalous neon appears together with enhanced ${ }^{40} \mathrm{Ar} /{ }^{36} \mathrm{Ar}$ isotope ratios, which is a strong argument in favour of a MORB component, but not of a solar origin. Confirmation of the above interpretation comes from the ${ }^{20} \mathrm{Ne} /{ }^{22} \mathrm{Ne}-{ }^{4} \mathrm{He} /{ }^{3} \mathrm{He}$ diagram (Fig. 2), where Kilauea data ${ }^{1}$ fill the gap between Loihi and MORB. Both data sets show alignments with the same negative slopes, which can be quantitatively attributed to a single stage mass fractionation.

Altogether, some of the data in ref. 1 can be explained by mixing combined with mass fractionation, but most of them are indeed isotopically atmospheric. The authors' conclusion that primordial terrestrial neon was isotopically solar is entirely based on their poorly defined $L-K$ line, and is therefore not strongly supported by the data, which, conversely, show clear mass fractionation effects which Honda et al. unfortunately did not discuss.

Ph. SARDA

Laboratoire de Géochimie et Th. STAUDACHER Cosmochimie,

Institut de Physique du Globe de Paris

Tour $14,3^{e}$ étage,

4 place Jussieu, 75252 Paris Cedex 05, France

HONDA ET AL. REPLY - Sarda and Staudacher argue that variation in observed neon isotope ratios in the Hawaiian samples could be attributed to recent isotope fractionation along paths parallel to the mass fractionation line (mfl), labelled $\mathrm{mdl}$ in Fig. 1. However, they disregard the fact that isotope frac- tionation must be accompanied by concomitant element fractionation. As we clearly stated ${ }^{1}$, the calculated ratio of nucleogenic ${ }^{21} \mathrm{Ne}$ to radiogenic ${ }^{4} \mathrm{He}$ in most of the Hawaiian samples listed in our table is close to the theoretical production ratio. Because the slope of the mixing line between solar and atmospheric components is not very different from that of the mfl, the amount of nucleogenic ${ }^{21} \mathrm{Ne}$ is effectively calculated as the deviation from the $\mathrm{mfl}$ line passing through the atmospheric composition. Thus, calculation of the nucleogenic ${ }^{21} \mathrm{Ne} /$ radiogenic ${ }^{4} \mathrm{He}$ ratio is essentially the same irrespective of which model is chosen to account for the high ${ }^{20} \mathrm{Ne} /{ }^{22} \mathrm{Ne}$ ratios in the Hawaiian samples. The similarity between the theoretical production ratio and the calculated nucleogenic ${ }^{21} \mathrm{Ne}$ /radiogenic ${ }^{4} \mathrm{He}$ ratio in the samples is regarded as strong evidence that there has been no substantial element fractionation between helium and neon. As isotope fractionation is likely to occur only under physical conditions more extreme than those leading to element fractionation, this apparent lack of element fractionation suggests that isotope fractionation of neon is unlikely to have occurred.

We have now shown ${ }^{6}$ that there are additional and systematic correlations between the isotope compositions of neon and helium in terrestrial samples, including basaltic glasses from both plume (Hawaii) and mid-ocean-ridge situations, gas samples from the continental hydrothermal system associated with the Yellowstone hotspot and ancient Zaire diamonds. The solar hypothesis successfully accounts for helium and neon isotope sytematics of all available samples, irrespective of sample type, tectonic setting and age, providing us with additional confidence that the Earth's primordial noble-gas composition contains a solar component.

M. HONDA

I. MCDOUGALL

D. B. PATTERSON

A. DOULGERIS

Research School of Earth Sciences,

The Australian National University,

GPO Box 4,

Canberra, ACT 2601,

Australia

US Geological Survey,

D. A. Clague

Menlo Park,

California 94025, USA

1. Honda, M. et al. Nature 349, 149-151 (1991).

2. Sarda, Ph., Standacher, Th. \& Allègre, C. J. Earth planet Sci. Lett. 91, 73-88 (1988).

3. Staudacher, Th., Sarda, Ph. \& Allègre, C. J. Chem. Geol 89, 1-17 (1990).

. Kurz, M. D. et al. Geochim. Cosmochim. Acta. 51, 2905-2914 (1987).

5. Kurz, M. D. et al. Earth planet. Sci. Lett. 66, 388-406 (1983).

6. Honda M., McDougall I., Patterson D. B., Doulgeris A. \& Clague D. A. Lunar planet. Sci. XXII, 587-588 (1991) 